\title{
Optimization of Near Infrared Cured Polyester/Melamine Coil Coatings by the Use of Near Infrared Absorbing Primers
}

\author{
Cathren E. Gowenlock, Ian Mabbett and David A. Worsley
}

\begin{abstract}
Near Infrared (NIR) radiative curing is a fast thermal curing technology used to cure 25 micron polyester melamine industrial coil coatings on galvanized steel substrate. The cure is optimized when the top coat is relatively transparent to NIR and with most NIR absorption taking place at the substrate surface. In this study UV/Vis/NIR spectroscopy and lab scale curing trials with a white top coat were used to show that absorption in the bottom most layers of the coating system can be taken a stage further by the addition of NIR absorbing pigment to the primer layer. The results show energy efficiency benefits for NIR cure by increasing the amount of incident radiation absorbed without altering the colour properties of the coating. A similar smaller benefit is also evident with a conventional convection cure.
\end{abstract}

Index Terms-Coil coating, fast cure, melamine, Near Infrared, polyester

\section{INTRODUCTION}

Coil coating is a continuous roll to roll process which deposits an organic coating onto a substrate, frequently galvanized steel [1]. Interest in improved efficiency in the coil coating industry has led to the introduction of Near-Infra-Red (NIR) curing technology and has enabled polyester coatings produced for the exterior roofing and cladding market to be manufactured at increased line speeds and with reduced oven lengths [2]. These coatings are formed from thermosetting polyester resins, commonly cross-linked with hexamethoxymethylmelamine.

NIR curing employs light between 800 and 1500 nm, where most organic compounds absorb minimally. Conversely, metals absorb strongly in this region. Consequently NIR rapidly heats metals, and the high energy density cure results in 25 micron polyester coil coatings curing in times of less

Manuscript received March 08, 2013. This work was supported by the ESF via WEFO funding the STRIP project, and with technical input, supply of materials and hosting of researchers at their research labs by Tata Steel Colors and BASF Coatings Ltd.

C. E. Gowenlock is with SPECIFIC, College of Engineering, Swansea University, Swansea SA12 7AX, UK ( phone: +44 1792 606604; fax: +44; email:625992@ swansea.ac.uk).

I. Mabbett is with SPECIFIC, College of Engineering, Swansea University, Swansea SA12 7AX, UK ( i.mabbett@ swansea.ac.uk).

D. A. Worsley is with SPECIFIC, College of Engineering, Swansea University, Swansea SA12 7AX, UK ( d.a.worsley@ swansea.ac.uk). than 10 seconds. This contrasts with cure times of 30 - 45 seconds in a conventional convection oven.

NIR absorbing pigments have been used to improve surface hydrophobicity of NIR cured sol gel coatings by creating a micro structured surface [3]. Previous research has also suggested that NIR absorbing pigments may boost the energy efficiency of NIR cure with polyester coil coatings [4]. However, more recent research has concluded that absorbing pigments may not always work since if too much energy absorption occurs in the top few microns of the cross-linking polyester coating, film formation may occur before full removal of solvents [5].

NIR curing can hence be optimized when the polyester top coat is relatively transparent to NIR and with the galvanized steel substrate absorbing most of the radiation [5]. $\mathrm{TiO}_{2}$ is a strong reflector of NIR radiation, whilst carbon black is a strong absorber. White coatings are hence easier to cure than their darker counterparts, having a wider cure window and being less prone to solvent boil, a phenomenon where a micro blistered surface results due to the top layers of the coating curing before sufficient solvent has been removed underneath.

In the work presented here, the benefits of absorption occurring in the bottom most layers of the coating system is taken a stage further by tinting the primer layer of the coating system with a NIR absorbing material (Special Black ${ }^{\circledR} 100$ carbon black).

This tinted primer is applied to lab samples of hot dip galvanized steel substrate (HDG) and then over coated with a white polyester top coat paint and NIR or convection cured. Similar lab samples are made substituting the tinted primer for a standard primer.

Spectroscopic studies and examination of the heat profiles of these samples show that a tinted primer could provide scope industrially to increase the energy efficiency of NIR cure, by increasing the amount of incident radiation absorbed without altering the color properties of the coating. Moreover, a smaller increase in energy efficiency can also be observed in a convection oven cure. 


\section{EXPERIMENTAL}

\section{A. Coatings preparation}

The primer used in this work was chromium free aqueous resin dispersion of combined pretreatment-primer, supplied by Henkel. Tinted primer was formulated by the addition of weighed quantities of Special Black ${ }^{\circledR} 100$ carbon black to the pretreatment-primer, dispersed with the aid of a magnetic stirrer. A Hegman gauge was used to check pigment agglomerates were less than 15 microns in size.

The white polyester melamine paint was a near commercial formulation modified slightly for NIR fast cure applications. Pigment agglomerations were milled to below 15 microns and checked with a Hegman gauge. Specification was between 40$50 \%$ gloss at $60^{\circ}$ and viscosity checked to take $65-70$ s to drain a DINN 4 flow cup at $21^{\circ} \mathrm{C}$. The formulation contained $34.54 \% \mathrm{w} / \mathrm{w}$ polyester resin, $24.87 \% \mathrm{w} / \mathrm{w}$ paint solvent, $25.21 \% \mathrm{w} / \mathrm{w} \mathrm{TiO}_{2}$ and $15.38 \% \mathrm{w} / \mathrm{w}$ other additives. Further detail of the formulation cannot be given for proprietary reasons.

\section{B. Spectroscopy}

UV-Vis-NIR total reflectance spectra were recorded using a Perkin Elmer Lambda $750 \mathrm{~S}$ high performance spectrophotometer equipped with a $60 \mathrm{~mm}$ integrating sphere; total reflectance being the sum of the specular and diffuse components of the sample reflectance. Total reflectance spectra were recorded of the HDG steel substrate, and also the HDG substrate coated with either primer or primer plus top coat.

Twenty micron paint free films were formed by coating onto PTFE coated steel and curing in a Mathis convection oven. UV-Vis-NIR transmission spectra of these free films were recorded using a Perkin Elmer Lambda 750S high performance spectrophotometer.

\section{Colour measurement}

Colour measurements were made on cured panels of HDG steel substrate coated with primer and white polyester top coat, using the CIELAB 1976 colour scale and a Datacolor Spectraflash 600 spectrophotometer.

\section{Convection and NIR cure}

Panels of HDG steel substrate for convection and NIR curing studies were coated with primer and white top coat using wire wound draw down bars, according to ASTM D4147. Primer thickness was $5 \mu \mathrm{m}$. Once the primer was cured, the panels were then over coated with $60 \mu \mathrm{m}$ wet films to give $20 \mu \mathrm{m}$ dry film thicknesses of white polyester topcoat. Panel sizes for NIR curing trials were $210 \mathrm{~mm}$ x $150 \mathrm{~mm}$ x $0.43 \mathrm{~mm}$, whilst for convection curing were $210 \mathrm{~mm} \times 100 \mathrm{~mm}$ $\mathrm{x} 0.50 \mathrm{~mm}$.

Cure profiles for the panels were measured using thermocouples and an Omega TC-08 data logger. Thermocouples were K-type, spot welded to the middle of the back of the substrate panels. The same panels were used in the spectroscopic work described in Section B.

Convection cure trials were carried out using a Mathis D70 convection oven set to $355^{\circ} \mathrm{C}$ with an oven dwell time of 30 seconds. Panels were assessed as cured by reaching a peak metal temperature (PMT) of between 216 and $232^{\circ} \mathrm{C}$, the polyester specification range for full cure at $20 \mu \mathrm{m}$ dry coating thickness. The coil coating industry commonly uses the resistance of a coating to a solvent rub test to indicate cure commonly the survival of the coating to double rubs with a 2butanone (methyl ethyl ketone or MEK) soaked cloth. A polyester coil coating that survives 100 double rubs with 2butanone before the substrate becomes visible is generally taken within the industry as an indication of adequate cure, and this 'MEK double rub test' was applied to the panels.

NIR cure trials were carried out using an AdPhos Coil Technicum, a lab based NIR oven. The AdPhos equipment is set up to simulate the NIR cure of industrial polyester coil coatings. This fast curing technique requires three oven temperature zones to cure 20 micron polyester melamine coatings; the first to rapidly heat the sample to a temperature where solvents will be removed, the second with more moderate temperature rise so that solvent removal can complete, and the third to rapidly heat to polyester cross linking temperature. The AdPhos Coil Technicum consists of a mobile sample holder which carries the wet coated substrate panel under banks of tungsten halogen lamps providing the NIR radiation. These lamps have variable power settings to achieve the desired oven temperature profile.

\section{RESULTS AND DISCUSSION}

\section{A. Spectroscopic studies}

The combined pretreatment primer layer used in this work is largely transparent to NIR radiation as is shown in Fig. 1. Transmission through a 5\% w/w carbon black tinted primer layer is however almost negligible as carbon black absorbs strongly over all wavelengths of the visible and NIR range.

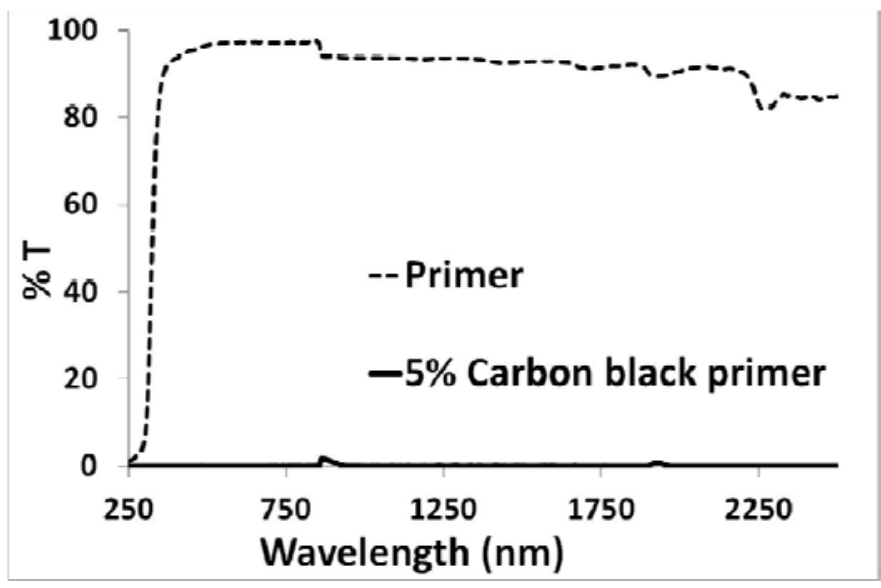

Fig. 1. Comparison of UV-Vis-NIR transmission spectra of free standing primer films. 
The noticeable feature of the UV-Vis-NIR total reflectance spectra of HDG steel substrate shown in Fig. 2 is a peak associated with $\mathrm{Zn}$ absorption around $1000 \mathrm{~nm}$ which is within the region of maximum NIR emission from the tungsten halogen NIR source. When the NIR transparent primer layer is coated onto this HDG substrate, only a modest decrease in absorption is seen in the reflectance spectra in Fig. 2. This is consistent with the primer layer being highly transparent; a high proportion of the radiation is making its way through the primer to give the characteristic $\mathrm{Zn}$ absorption peak at around $1000 \mathrm{~nm}$. A pretreatment-primer layer tinted with carbon black however, shows extremely low transparency to NIR radiation, absorbing most of the incident radiation as shown by the solid line in Fig. 2 .

Recent work has shown that the optimum scenario for polyester coating cure is when the top coat has some level of transparency to NIR, allowing the HDG surface beneath the top coat to absorb NIR and heat the substrate. This will have the effect of driving solvents upwards through the coating system and allowing them to escape more easily [5]. This is advantageous as early film formation associated with absorption occurring in the first few microns of the top coat is prevented, a scenario which leads to 'solvent boil' i.e. fine surface micro blisters appearing, caused by solvent bursting through an already skinned surface.

Substituting a relative transparent primer for the relatively opaque tinted primer has therefore had the effect of moving the locus of maximum NIR absorption from the HDG surface to the tinted primer layer, whilst at the same time increasing the amount of absorption that is taking place. This would suggest that a coating system with a tinted primer may require a lower powered NIR source, as less incident radiation is being wasted in reflection.

Clear polyester has a high degree of transparency to NIR radiation, as shown by the transmission spectrum of the free standing polyester film in Fig. 3. The same figure also shows that white polyester (pigmented with titanium dioxide) still allows a sizeable amount of NIR radiation to pass through.

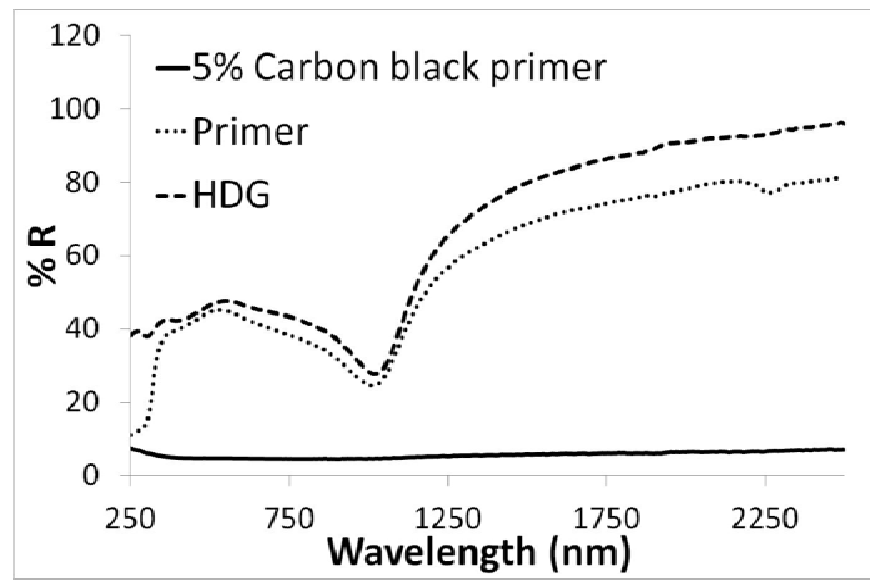

Fig. 2. UV-Vis-NIR reflectance spectra of HDG panels coated with primer.

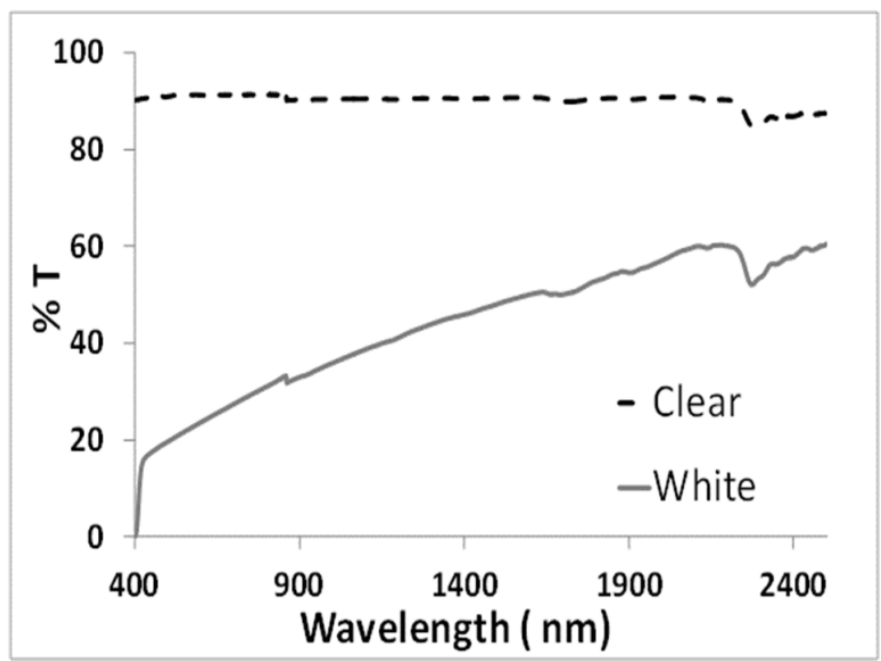

Fig. 3. Comparison of Vis-NIR transmission spectra of free standing polyester films

White top coats were then applied over standard and tinted pretreatment-primers coated on HDG steel substrate. These showed similar reflectance spectra in the UV and visible region, as shown in Fig. 4 where the visible region is highlighted by the shaded area. In the NIR region, the tinted pretreatment-primer sample shown in Fig. 4 shows considerably less reflectance than the standard pretreatment primer sample indicating that some NIR radiation is able to penetrate through the top coat and be absorbed by the carbon black underneath.

The similar visible reflectance spectra in Fig. 4 concur with CIELAB 1976 delta E* colour scale measurements made with the white top coat panel under coated with untinted primer as the standard. This shows a delta $E^{*}$ value of less than 1.1 for $5 \%$ absorbing pigment in the primer layer. Delta $\mathrm{E}^{*}$ of 1.0 is commonly taken as barely perceptible to the human eye, and this can be confirmed to be the case.

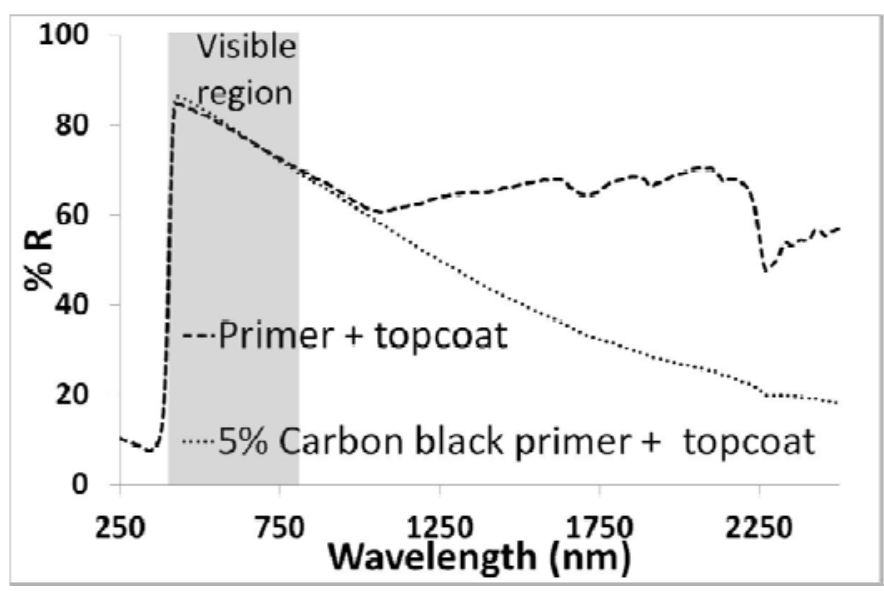

Fig. 4. UV-Vis-NIR reflectance spectra of HDG coated with primer and 20 microns white top coat.

\section{B. Cure profiles}

Panels with white top coat were then cured in a NIR oven, each sample being cured at the same power setting. An 18\% 


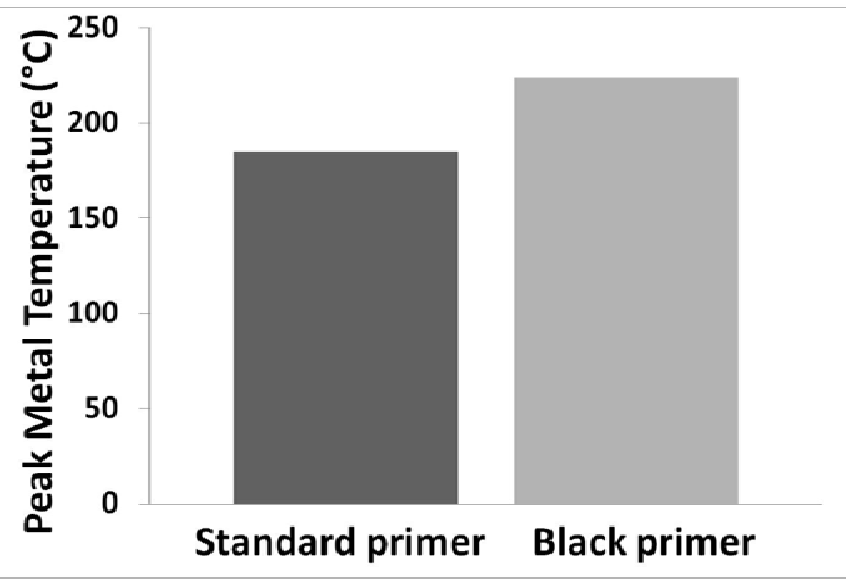

Fig. 5. PMTs reached by panels with different primers with identical NIR curing conditions.

lower peak metal temperature (PMT) was observed when a standard pretreatment primer was used rather than a carbon black tinted primer, as shown in Fig. 5 . As a consequence, this method offers the opportunity to reduce radiation intensity during NIR cure to obtain the same PMT and hence to reduce energy consumption.

Similar experiments with panels cured in a convection oven also showed lower PMTs with a standard pretreatment primer. In this case black body emission from the walls of the convection oven produce a 5\% lower PMT during cure when standard primer is used compared to $5 \%$ carbon black tinted primer.

\section{Interaction of NIR photons with the coating system}

Previous work has proposed mechanisms for NIR photon interactions with lighter colored less strongly absorbing coating systems and with darker more highly absorbing coating systems [5]. Proposed mechanisms for photon interactions with a paint system incorporating a NIR absorbing primer are deduced from the spectral information in Figs. 1-4 and given in Fig. 6. Mechanisms $A$ and A' show photon absorption by the top coat, with better depth penetration at A'. As white coatings are largely transparent to NIR photons, mechanism $\mathrm{B}$ (absorption of photons by the primer layer) is likely to predominate. Mechanism C (photons reflected at the air/coating surface) will also be significant for a white top coat.

Benefits of tinting the primer layer are that mechanism B will result in heating of the top coat by conduction from the primer, causing the bottom most layers of the top coat closest to the primer to heat up very quickly. This will drive solvents upwards from the region in which they have furthest to diffuse. Lower regions of the coating will cure before the upper regions; methanol, the resin cross linking by-product will escape more easily and solvent boil will be minimized.

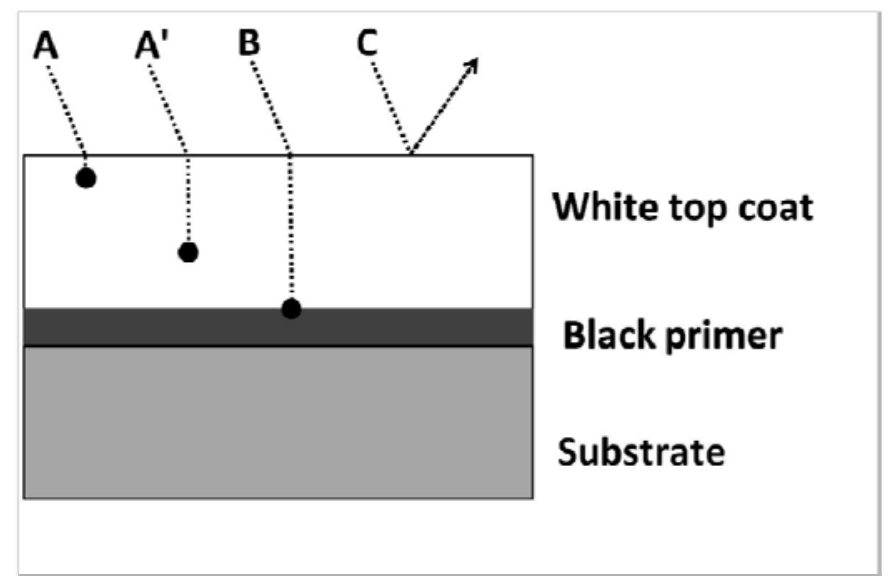

Fig. 6. Proposed mechanisms for photon interaction with coating system.

\section{CONCLUSION}

This work is of importance for the manufacture of coil coatings cured by NIR or convection ovens because it provides scope for improved energy efficiency by increasing the amount of incident radiation absorbed without altering the color properties of the coating. The increased NIR absorbance of the primed surface allows for the combination of increased line speeds and reduced oven power settings to reach a given PMT value.

\section{ACKNOWLEDGMENT}

The authors would like to acknowledge the support of the ESF via WEFO for funding the STRIP project and Tata Steel Colors and BASF Coatings Ltd for technical input, the supply of materials and hosting the researcher (CG and IM) at their research labs.

\section{REFERENCES}

[1] C. Perruchot, J. F. Watts, C. Lowe and G. Beamson, "Characterisation of the curing temperature effects on polyester systems by angle-resolved XPS (ARXPS),” Int. J. Adhes. Adhes., vol. 23(2), pp. 101-113, 2003.

[2] K. K. O. Bar, "How to save energy costs with the NIR-technology in coil coating processes," Steel Grips, vol. 3, pp. 225-228, 2006.

[3] R. Subasri, C. S. Madhav, K. R. C. Somaraju, G. Padmanabham, "Decorative, hydrophobic sol-gel coatings densified using near-infrared radiation,” Surf. Coat. Technol., vol. 206, pp. 2417-2421, 2012.

[4] R. Knischka, U. Lehmann, U. Stadler, M. Mamak, J. Benkhoff, "Novel approaches in NIR curing technology,” Prog. Org. Coat., vol. 64 (2-3), pp. 171-174, 2009.

[5] I. Mabbett, J. Elvins, C. Gowenlock, P. Jones and D. Worseley, "Effects of colour on near infrared cured polyester/melamine coil coatings," Prog. Org. Coat., to be published

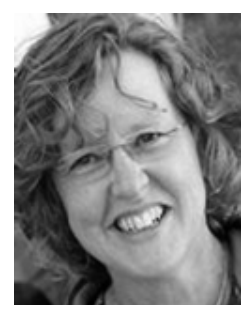

Cathren Gowenlock obtained her BSc chemistry from University of York, UK in 1980 and MSc analytical chemistry from University of Bristol, UK in 1982.

She has previously worked as a Scientific Assistant and Operational Research Officer with British Gas, UK. Wishing to return to academia, she is now an Engineering Doctorate Research Engineer at Specific, Swansea University, UK, looking at fast radiative curing of organic coatings.

Ms. Gowenlock is a Member of the Royal Society of Chemistry. 